\title{
PRODUÇÃO DE ESTUDOS EM ENFERMAGEM SOB O REFERENCIAL DA FENOMENOLOGIA
}

\author{
PRODUCTION OF STUDIES IN NURSING UNDER THE \\ PHENOMENOLOGY REFERENTIAL
}

\section{PRODUCCIÓN DE ESTUDIOS EN ENFERMERÍA BAJO EL INDICE DE REFERENCIA DE LA FENOMENOLOGÍA}

\author{
Denise do Nascimento Esquivel ${ }^{1}$ \\ Gilberto Tadeu Reis da Silva ${ }^{2}$ \\ Maria Olivia Medeiros ${ }^{3}$ \\ Natália Rosas Batista Soares ${ }^{4}$ \\ Virginia Crispina de Oliveira Gomes \\ Simone Teixeira da Luz Costa ${ }^{6}$
}

\begin{abstract}
Objetivo: caracterizar a produção de estudos gerada pela pós-graduação e conduzidos segundo o referencial da fenomenologia. Metodologia: pesquisa documental, retrospectiva, de natureza quantiqualitativa, utilizando versão eletrônica dos Catálogos do Centro de Estudos e Pesquisas em Enfermagem (CEPEn) da Associação Brasileira de Enfermagem (ABEn) publicados no período compreendido entre 2003 e 2013. Resultados: identificado número considerável de estudos desenvolvidos por pesquisadores da área de enfermagem segundo o pensamento filosófico de Alfred Schutz e Martin Heidegger, nos quais a investigação dos modos possíveis do cuidar abre perspectivas para um novo olhar do cuidado na enfermagem. Conclusão: o quantitativo das publicações gerado pela pós-graduação reflete o interesse pelo referencial teórico, assim como a busca profissional pelo aprimoramento do entendimento do ser por meio dos postulados fenomenológicos.
\end{abstract}

Descritores: Enfermagem; Fenomenologia; Cuidar.

Objective: to characterize the production of studies generated by graduate students and conducted in accordance with the phenomenology referential. Method: a retrospective documentary research, of a quantitative and qualitative nature, using the electronic version of the catalogues of the Center for Studies and Research in Nursing (CEPEn) of the Brazilian Association of Nursing (ABEN), published in the period 2003-2013. Results: a considerable number of studies were identified, carried out by researchers of the nursing area according to the philosophical thought of Alfred Schutz and Martin Heidegger, in which the investigation of possible ways of caring opens perspectives for a new look into nursing care. Conclusion: the quantitative of the publications generated by the graduate students reflects the interest for the theoretical referential, as well as the professional quest for improving the understanding of the buman being through underlying phenomenological means.

Descriptors: Nursing; Phenomenology; Care.

\footnotetext{
Enfermeira. Especialista em Enfermagem Intensiva pela Universidade Federal da Bahia. Salvador, Bahia, Brasil. deniseesquivel83@yahoo.com.br

Pós-Doutor em Ensino em Ciências da Saúde pela Universidade Federal de São Paulo (Unifesp). Professor Titular da Universidade Federal da Bahia. Líder do Grupo de Pesquisa: Grupo de Estudos em Pesquisa em Administração do Serviço em Enfermagem. Salvador, Bahia, Brasil. gilberto.tadeu@ufba.br

Enfermeira. Especialista em Cardiologia e Hemodinâmica pela Escola Bahiana de Medicina e Saúde Pública. Docente da Escola Bahiana de Medicina e Saúde Pública. Salvador, Bahia, Brasil. poppmedeiros@yahoo.com.br

4 Enfermeira. Especialista em Auditoria em Serviços de Saúde pela Universidade Castelo Branco. Especialista em Enfermagem Intensiva pelo Centro de Pós-graduação São Camilo. Membro do Grupo de Estudos em Pesquisa em Administração do Serviço de Enfermagem. Salvador, Bahia, Brasil. nataliarosas.00@gmail.com

Enfermeira. Mestre em Enfermagem pela Universidade Federal da Bahia. Especialista em Enfermagem do Trabalho pela Faculdade de Tecnologia Internacional. Salvador, Bahia, Brasil.viwcris@hotmail.com

Enfermeira. Especialista em Enfermagem Intensiva pela Universidade Federal da Bahia. Especialista em Cardiologia e Hemodinâmica pela Escola Bahiana de Medicina e Saúde Pública. Membro do Grupo de Estudos em Pesquisa em Administração do Serviço em Enfermagem. Mestranda em Ensino na Saúde pela Universidade Federal de São Paulo (Unifesp). Salvador, Bahia, Brasil. simonecostal9@yahoo.com.br
} 
Objetivo: caracterizar la producción de los estudios generados por graduados y realizados de acuerdo con el referencial de la Fenomenología. Método: investigación documental retrospectiva, de naturaleza cuantitativa y cualitativa, utilizando la versión electrónica de los catálogos del Centro de Estudios e Investigación en Enfermería (CEPEn) de la Asociación Brasileña de Enfermería (ABEN), publicados en el período 2003-2013. Resultados: se identificó un número considerable de estudios llevados a cabo de acuerdo con el pensamiento filosófico de Alfred Schutz y Martin Heidegger por investigadores del área de enfermería, en el que la investigación de las posibles formas de cuidar abre perspectivas para una nueva mirada en el cuidado de enfermería. Conclusión: el cuantitativo de las publicaciones generadas por graduados reflete el interés por el referencial teórico, así como la búsqueda profesional por el perfeccionamiento del entendimiento del ser por medio de los postulados fenomenológicos.

Descriptores: Enfermeria; Fenomenología; Cuidado.

\section{Introdução}

A vivência de cuidar pode ser considerada uma forma de garantir a manutenção da existência. O homem requer cuidado para se manifestar de maneira plena como ser no mundo e, portanto, é possível assegurar que o cuidado é inerente à sua existência. Desde a origem da humanidade, a ação de cuidar faz-se presente, como maneira de viver ou de se relacionar no mundo ${ }^{(1)}$.

O processo de cuidar é concebido como essência da Enfermagem e dirige-se às várias esferas da vida da pessoa, incluindo sua necessidade de atenção, amor, respeito e compreensão. Nesse sentido, o referencial da fenomenologia pode contribuir para a condução de investigações com o intuito de otimizar a maneira como se cuida, pois possibilita a apreensão do homem enquanto ser existente no mundo, vivenciando facticidades, o que afeta o seu modo de se relacionar com o mundo e com todas as coisas nele envolvidas. Os enfermeiros, quando optam por desenvolver estudos sob esse referencial, podem fundamentar-se nas distintas vertentes de pensamento, o que permite a apreensão da dimensão ontológica, existencial, do homem a ser cuidado ${ }^{(1)}$.

O cuidado, finalidade da assistência de Enfermagem, é melhor gerido quando contempla as múltiplas dimensões do homem a quem se dirige. A tendência é voltar-se para pessoas conscientes e livres, e não para seres anônimos. Compreender que a essência do cuidado só é alcançada pelo acesso à experiência da pessoa cuidada, apreendendo sua realidade vivenciada, possibilita que o profissional visualize novas formas de entender o outro, dele cuidando sob novos parâmetros assistenciais ${ }^{(2)}$.

No plano das práticas é possível designar por Cuidado uma atitude terapêutica que busque ativamente seu sentido existencial. É a conformação humanizada do ato assistencial, distinguindo-se daquelas práticas que, por razões diversas, não visam ampliar e flexibilizar o uso das tecnociências da saúde. Embora o Cuidado, na filosofia heideggeriana, não diga respeito ao cuidar ou descuidar no sentido operativo do senso comum, e nem em uma perspectiva estritamente médica, adota-se esse termo como uma designação de uma atenção à saúde imediatamente interessada no sentido existencial da experiência do adoecimento, físico ou mental e, por conseguinte, também das práticas de promoção, proteção e recuperação da saúde ${ }^{(3)}$.

Ao abordar o Cuidado, geralmente se faz referência a um conjunto de princípios e estratégias que norteiam, ou devem nortear a relação entre um sujeito, o paciente, e o profissional de saúde que o atende. Construir um cuidado desse tipo é tão importante quanto investir na reflexão e transformação relativas das características das interações interpessoais nos atos assistenciais e com base neles; é debruçar-se continuamente sobre as raízes e os significados sociais dos adoecimentos em sua condição de obstáculos coletivamente postos a projetos de felicidade humana e, de forma articulada, da disposição socialmente dada das tecnologias e dos serviços disponíveis para sua superação. É como se aquele projeto, 
revalorizado, reconhecido, pudesse ser retomado em um novo plano, re-significando tudo à sua volta, inclusive, e especialmente, o cuidado de $\mathrm{si}^{(3)}$.

Heidegger aponta positivamente para o fato de que o ser humano é um estar lançado no mundo numa reconstrução constante de si mesmo e desse mundo, elucidada pela ideia de Cuidado. Será justo assumir que as práticas de saúde, como parte desse estar lançado, tanto quanto dos movimentos que o reconstroem, também se elucidam como Cuidado ${ }^{(3)}$. Entretanto, para alcançar esta magnitude do cuidado, é necessário compreender a dimensão existencial do ser humano, bem como os significados da sua experiência vivida ou daquele que oferece seus cuidados a outrem. O pensar fenomenológico permite alcançar essa compreensão por meio de descrições das experiências vividas pelos sujeitos ${ }^{(1)}$.

Neste contexto de lidar com a esfera existencial da pessoa que cuida e daquela que é cuidada, a fenomenologia traz uma importante e fundamental contribuição, na medida em que propõe o resgate da subjetividade como possibilidade de alcançar a essência do outro. Propõe-se a descrever os fenômenos tal como ocorrem, olhando as coisas como se manifestam e, assim, desvelando sua essência. Tal perspectiva implica a coexistência e a participação.

A fenomenologia apresenta-se como ciência descritiva, rigorosa, concreta, que mostra e explicita preocupação com a essência do vivido. É ainda uma ciência do possível, isto é, refere-se à possibilidade enquanto modo de ser da existência humana, que possui projetos existenciais ${ }^{(4)}$.

Conforme relatado na literatura, a fenomenologia nos convoca a retomar o caminho qualitativo da existência, a redescobrir o sentido global do existir no mundo, o sentido de ser humano no mundo, o sentido de nossa existência. Assim, não podemos abandonar nem a razão nem a técnica, mas sim recuperar o sentido do ser, da existência humana, do ser humano no mundo ${ }^{(5)}$.

É ressaltada, na fenomenologia, a importância do sujeito no processo de construção do conhecimento, procurando interrogar a experiência vivida e o significado que o sujeito lhe atribui. Isto implica em procurar não priorizar o objeto e/ou sujeito, mas centrar-se na relação sujeito-objeto-mundo, buscando a indissociação entre eles. A fenomenologia, na perspectiva de Husserl, possibilita ao pesquisador o acesso à consciência humana, a volta às coisas mesmas, às essências, o que significa chegar às verdades, à realidade desprovida de estereótipos ou a pressupostos em relação ao fenômeno pesquisado. Autores referem-se à fenomenologia como o discurso esclarecedor do que se mostra oculto e tem o sentido de des-velar (tirar o véu, desocultar). Tem como objeto de estudo a realidade vivida pelos sujeitos ${ }^{(6)}$.

$\mathrm{Na}$ área de enfermagem, em especial, o campo de investigação passou por um redirecionamento por meio do qual se abriram outras possibilidades de investigação. As enfermeiras ousaram recorrer a novas metodologias, com o objetivo de dar continuidade à construção de um corpo de conhecimentos, agora contemplando novos objetos de investigação. Esse redirecionamento se dá em consonância com a crítica mundial feita ao enfoque positivista por parte das ciências sociais ${ }^{(7)}$.

O pensamento positivista, com sua observância à quantificação, à objetividade e ao estabelecimento de relações entre fatos estatisticamente significantes, encerrava a análise das realidades precisamente no ponto onde deveria iniciá-la. Esses momentos de ruptura epistemológica são fundamentais para o emergir de novas concepções científicas por meio do reconhecimento do cientista de que os paradigmas disponíveis não conseguem explicar um fenômeno ou um fato novo.

A natureza de determinados objetos de investigação e, fundamentalmente, a forma de visá-los possibilitou um avanço na construção de um corpo de conhecimentos em enfermagem, gerando um impulso e uma consolidação de novas formas de pesquisar. Com a incorporação desse primeiro corpo de conhecimentos científicos e de forma interligada aos avanços da ciência, e também após novas incorporações na esfera tecnológica, além de um descortinamento da própria concepção de ciência, novos objetos foram se mostrando aos 
enfermeiros como carentes de investigação. Até mesmo um objeto já estudado foi se revelando sob outras óticas, diferentes perspectivas.

Assim, enfermeiros preocupados com as relações interpessoais no contexto hospitalar, em toda sua abrangência e considerando os codeterminantes gerados por profundas modificações decorrentes da intensificação tecnológica, voltam-se para ideias que venham contemplar a perspectiva do sujeito a quem o cuidado é prestado. Nesse sentido, emergem a linguagem, a relação dialógica, a intersubjetividade como componentes essenciais na produção de um novo conhecimento. A fenomenologia apresenta-se, então, como um referencial para fundamentar investigações pertinentes ao cotidiano de trabalho do enfermeiro, quando se fazem presentes situações de dor, sofrimento, perdas, relações empáticas, rejeição e conflitos.

Nesse contexto, dissertações de mestrado e teses de doutorado passaram a ser realizadas, a partir da década de oitenta, segundo o referencial metodológico da fenomenologia, trazendo a dimensão existencial do sujeito a quem o cuidado é oferecido.

Este estudo tem como objeto investigado a produção acadêmica de enfermagem gerada pela pós-graduação e conduzida segundo a metodologia de investigação fenomenológica.

Diante do exposto, emergiu a seguinte questão para o presente estudo: Qual a produção de estudos gerada pela pós-graduação e conduzida segundo o referencial da fenomenologia? O objetivo é caracterizar a produção de estudos gerada pela pós-graduação e conduzida segundo o referencial da fenomenologia. Serão priorizados os seguintes aspectos: ano e tipo das publicações, área do cuidar, unidade federativa da instituição, instituição, programa de pós-graduação e referencial filosófico adotado.

Destaca-se a importância deste estudo, uma vez que a identificação de investigações que adotem este referencial sinaliza a associação Fenomenologia-Enfermagem-Cuidado, que concorrem para o alcance do cuidar em toda a sua magnitude. Além disso, busca descrever situações vividas, desvelar o que está encoberto e abrir caminhos para novas dimensões para o cuidado.

\section{Metodologia}

Trata-se de pesquisa documental, retrospectiva, de natureza quantiqualitativa, utilizando como fonte de informações os Catálogos do Centro de Estudos e Pesquisas em Enfermagem (CEPEn) da Associação Brasileira de Enfermagem (ABEn) publicados no período de 2003 a 2013, disponíveis em versão eletrônica. Estudo semelhante foi realizado com recorte temporal de 1987 a 2004. Então, houve interesse em caracterizar a produção sobre a temática na década seguinte, com recorte entre 2003 e $2013^{(8)}$. O critério de inclusão foi a existência da palavra "fenomenologia" nos resumos das teses ou dissertações. Não foi possível adotar a busca de trabalhos publicados por meio de descritores indexados, em virtude da indisponibilidade desses nos catálogos publicados até o ano de 2010. O critério de exclusão foi a duplicação do resumo.

A coleta de dados ocorreu nos meses de abril e maio de 2015 e foi orientada por um quadro, que possibilitou o agrupamento das seguintes informações: título do resumo, ano de publicação, programa de pós-graduação, unidade federativa das instituições de ensino, área do cuidado e referencial filosófico.

A análise dos estudos localizados nos catálogos do CEPEn foi realizada após a leitura de todos os resumos, por meio da técnica de leitura flutuante, que permitiu selecionar e caracterizar as produções conduzidas segundo a abordagem fenomenológica. De posse dos dados e das informações a respeito do número e da caracterização das produções envolvendo o objeto de pesquisa, prosseguimos para a análise dos dados obtidos nas leituras atentivas dos estudos, apreendendo também as áreas do cuidado por eles abordadas.

\section{Resultados e Discussão}

Entre 2003 e 2013 foram divulgados 5.886 estudos nos Catálogos de Teses e Dissertações do 
CEPEn. Dentre estes, 108 foram conduzidos segundo a abordagem fenomenológica. O Gráfico 1 exibe esse quantitativo de acordo com os anos em que foram publicados.

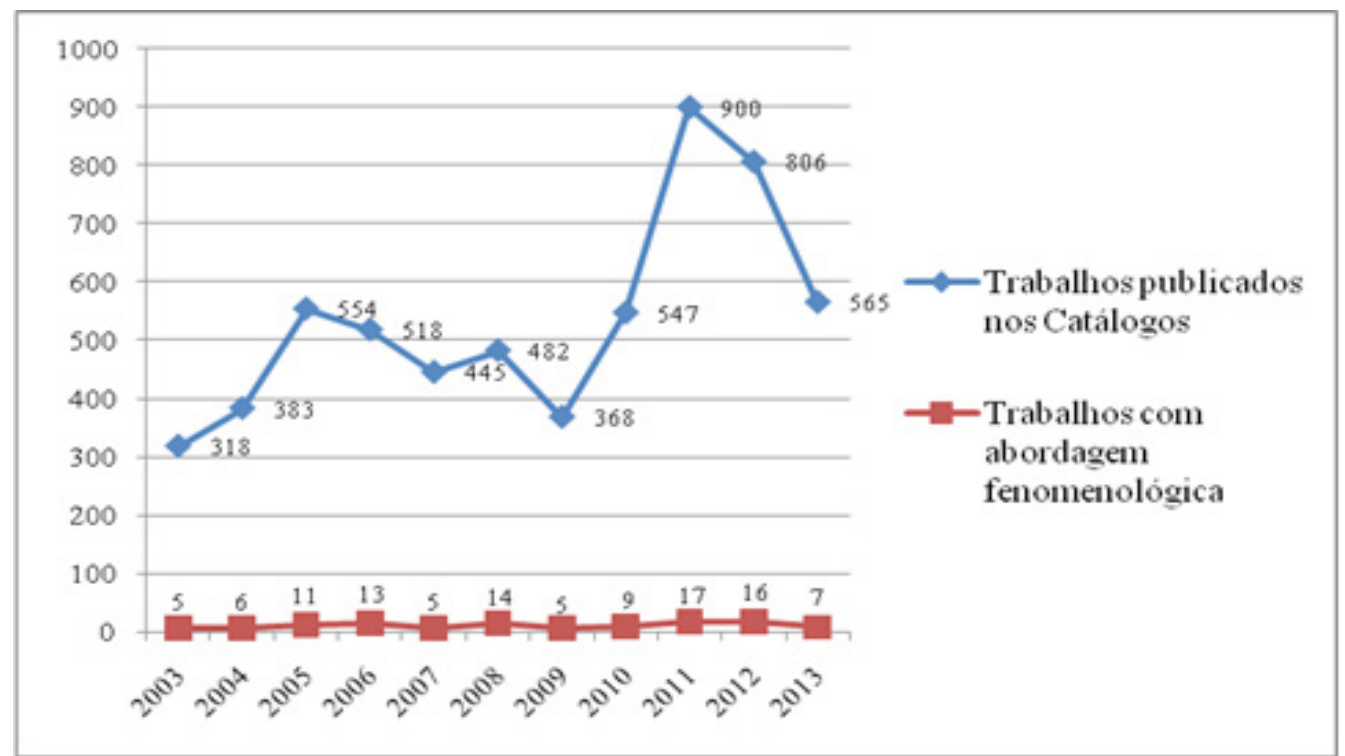

Gráfico 1 - Quantitativo de trabalhos publicados nos Catálogos do Centro de Estudos e Pesquisas em Enfermagem segundo abordagem fenomenológica e ano de publicação. Brasil, 2003-2013

Fonte: Elaboração própria.

Estatisticamente, o percentual de estudos à luz da fenomenologia, entre os anos de 2003 e 2013, corresponde a 1,8\%. Em 2006, estudo realizado na mesma fonte de informação, compreendendo o período de publicações de 1987 a 2004 , identificou que $14,8 \%$ estavam sob o referencial teórico da fenomenologia ${ }^{(9)}$. A comparação entre os resultados desse estudo de 2006 e os da pesquisa ora realizada mostra uma diminuição do número de estudos com abordagem fenomenológica. Pode-se deduzir que esse decréscimo no número de estudos deve-se ao fato de serem estudos que requerem tempo para serem concluídos.

Durante a coleta dos dados, foi observado que, até o ano de 2010, não havia descrição, nos Catálogos de Dissertações e Teses, das áreas de cuidado investigadas individualmente pelos pesquisadores, o que requereu a análise de cada resumo divulgado e/ou consulta das publicações em meio eletrônico para identificar tal informação.

As Tabela 1A e 1B relacionam os referenciais filosóficos utilizados e as áreas de cuidado respectivamente categorizadas. 
Tabela 1A - Correlação entre Áreas do Cuidar e Referencial Teórico Filosófico da Fenomenologia nas produções de enfermagem. Brasil, 2003-2013 ( $\mathrm{N}=108)$

\begin{tabular}{l|c|c|c|c|c}
\hline \multirow{2}{*}{$\begin{array}{l}\text { Áreas do cuidado/ } \\
\text { Referencial teórico }\end{array}$} & \multicolumn{5}{c}{ Categorias } \\
\cline { 2 - 6 } & $\begin{array}{c}\text { Saúde do } \\
\text { Adulto/Idoso }\end{array}$ & $\begin{array}{c}\text { Saúde } \\
\text { Mental }\end{array}$ & $\begin{array}{c}\text { Saúde da } \\
\text { Mulher }\end{array}$ & $\begin{array}{c}\text { Saúde do } \\
\text { Adolescente }\end{array}$ & $\begin{array}{c}\text { Saúde } \\
\text { Coletiva }\end{array}$ \\
\hline Alfred Schutz & 2 & 3 & 2 & 3 & 2 \\
Martin Heidegger & 3 & 2 & 7 & 2 & - \\
Maurice Merleau-Ponty & 2 & - & 5 & - & - \\
Maurice Merleau-Ponty/Paul Ricoeur & - & 1 & - & - & - \\
Joel Martins & - & - & - & - & - \\
Viktor Frankl/Giorgi (adptado) & - & - & - & - & - \\
Amadeo Giorgi & - & - & - & - \\
Paterson/Zderad & 1 & - & - & - \\
Edmund Husserl & - & - & - & - \\
Colaizzi & - & - & 1 & - \\
Yolanda Forghieri & - & - & - & - \\
Não Especificado & - & 3 & - & - \\
Total & 8 & 9 & 15 & - \\
\hline
\end{tabular}

Fonte: Elaboração própria.

Nota: Sinal convencional utilizado:

- Dado numérico igual a zero não resultante de arredondamento.

Tabela 1B - Correlação entre Áreas do Cuidar e Referencial Teórico Filosófico da Fenomenologia nas produções de enfermagem. Brasil, 2003-2013 ( $\mathrm{N}=108)$

\begin{tabular}{|c|c|c|c|c|c|}
\hline \multirow{2}{*}{$\begin{array}{l}\text { Áreas do cuidado/ } \\
\text { Referencial teórico }\end{array}$} & \multicolumn{5}{|c|}{ Categorias } \\
\hline & Oncologia & Pediatria & $\begin{array}{l}\text { Trabalho em } \\
\text { Enfermagem }\end{array}$ & $\begin{array}{c}\text { Ensino em } \\
\text { Enfermagem }\end{array}$ & $\begin{array}{l}\text { Cuidado } \\
\text { em Saúde }\end{array}$ \\
\hline Alfred Schutz & 1 & 5 & 8 & 9 & 10 \\
\hline Martin Heidegger & 3 & 3 & & 3 & 6 \\
\hline Maurice Merleau-Ponty & - & - & - & 2 & 1 \\
\hline $\begin{array}{l}\text { Maurice Merleau-Ponty/ } \\
\text { Paul Ricoeur }\end{array}$ & - & - & - & - & 3 \\
\hline Joel Martins & - & 1 & 1 & - & \\
\hline $\begin{array}{l}\text { Viktor Frankl/Giorgi } \\
\text { (adptado) }\end{array}$ & - & - & - & - & 2 \\
\hline Amadeo Giorgi & - & - & - & - & 1 \\
\hline Paterson/Zderad & - & - & - & - & - \\
\hline Edmund Husserl & 1 & - & - & - & - \\
\hline Colaizzi & - & - & - & - & - \\
\hline Yolanda Forghieri & - & 1 & - & - & - \\
\hline Não Especificado & - & - & 3 & 1 & 3 \\
\hline Total & 5 & 10 & 12 & 15 & 26 \\
\hline
\end{tabular}

Fonte: Elaboração própria.

Nota: Sinal convencional utilizado:

- Dado numérico igual a zero não resultante de arredondamento. 
Dos 108 trabalhos encontrados, destacam-se as publicações na área do cuidado em saúde, seguidas por ensino em enfermagem e saúde da mulher, ambas com a mesma frequência. Foram identificados 74 estudos desenvolvidos por pesquisadores da área de enfermagem, segundo o pensamento filosófico de Alfred Schutz e Martin Heidegger, nos quais a investigação dos modos possíveis do cuidar abre perspectivas para um novo paradigma de cuidado, para além do biológico, evidenciando a busca da enfermagem por aprimorar a assistência prestada.

Estudos conduzidos segundo outros referenciais, como Amadeo Giorgi, Paterson Zderad, Edmund Husserl, Colaizzi e Yolanda Forghieri, foram menos expressivos numericamente (5 estudos) no período da pesquisa, sendo 1 de cada referencial.

O referencial filosófico mais utilizado nas produções de pós-graduação pesquisadas corresponde à fenomenologia social de Alfred Schutz, com 45 estudos. A análise fenomenológica do filósofo traz uma proposta socioexistencial, na qual o ponto de partida é o individual, embora não se atenha a ele, chegando-se à compreensão do significado do cuidar numa dimensão social $^{(10)}$. Isso justifica a associação desta natureza filosófica a todas as áreas do cuidado, conforme apontam as Tabelas 1A e 1B.

Na Tabela 2 é possível notar maior número de publicações, nos cursos de Mestrado, com referencial filosófico de Alfred Schutz, seguido de Martin Heidegger e Maurice Merleau-Ponty.

Tabela 2 - Número de produções científicas segundo Referencial Filosófico e programas de pós-graduação. Brasil, 2003-2013

\begin{tabular}{l|c|c}
\hline \multirow{2}{*}{ Referencial Filosófico } & \multicolumn{2}{c}{ Programa de Pós-graduação } \\
\cline { 2 - 3 } & Mestrado & Doutorado \\
\hline Alfred Schutz & 30 & 15 \\
Martin Heidegger & 18 & 11 \\
Maurice Merleau-Ponty & 6 & 5 \\
Maurice Merleau-Ponty / Paul Ricoeur & 4 & - \\
Joel Martins & 2 & - \\
Viktor Frankl / Giorgi (adaptado) & 2 & - \\
Amadeo Giorgi & 1 & - \\
Paterson / Zderad & 1 & 1 \\
Edmund Husserl & 1 & - \\
Colaizzi & - & 3 \\
Yolanda Forghieri & 1 & 35 \\
Não Especificado & 7 & - \\
Total & 73 & \\
\hline
\end{tabular}

Fonte: Elaboração própria.

Nota: Sinal convencional utilizado:

- Dado numérico igual a zero não resultante de arredondamento.

No período de 2003 a 2013 foram publicadas, nos Catálogos do CEPEn, 30 dissertações e 15 teses com referencial filosófico de Alfred Schutz, 18 dissertações e 11 teses alicerçadas no referencial de Martin Heidegger e 6 dissertações e 5 teses pautadas nos princípios de Maurice
Merleau-Ponty. Martin Heidegger debruçou sua análise e reflexão sobre o sentido do ser em sua obra Ser e Tempo, possibilitando a compreensão do ser do ente com base na discussão sobre os modos de ser cotidiano, considerando a mundanidade e a temporalidade. Em sua visão 
antropológica, desvela a essência do homem e seu significado existencial, inferindo que a essência do homem depende de sua relação com o ser $^{(10)}$.

É possível fazer uma inferência condizente com o quantitativo das publicações em pós-graduação, o que reflete o interesse pelo referencial teórico, assim como a busca profissional pelo aprimoramento do entendimento do ser por meio dos postulados fenomenológicos.

Destacamos também que, em 10 resumos contidos nos Catálogos, não havia citação do filósofo que alicerçou a pesquisa. Se comparamos ao estudo realizado em $2006^{(8)}$, entre os anos de 1987 e 2004, verificamos o mesmo perfil quanto à liderança no número de produções embasadas, sequencialmente, em Schutz, Heidegger e Ponty, seguida de outros referenciais em menor número. Ressaltamos, portanto, a importância do referencial teórico de Alfred Schutz, entendendo que o cuidado de enfermagem pode ser considerado como uma ação social que tem como cenário o mundo da vida, no qual se estabelecem relações intersubjetivas que podem ser edificadas pelo enfermeiro nos diversos contextos em que atua $^{(11)}$.

Após a coleta dos dados, observamos também maior produção científica voltada à abordagem fenomenológica, desenvolvida por enfermeiros da região Sudeste do Brasil, com divulgação de 79 trabalhos, o que representa aproximadamente $73 \%$ do total analisado. A Universidade de São Paulo liderou a estatística, com 22 estudos de doutorado e 14 de mestrado, como evidencia a Tabela 3.

Destaca-se também que o número de instituições que divulgaram dissertações de mestrado nos Catálogos foi bastante superior em relação aos estudos de doutorado. A região Sudeste apresentou 10 universidades que disponibilizam cursos e programas de pós-graduação na modalidade stricto sensu, fato que pode ter contribuído para o resultado. O quantitativo expresso nessa região é consequência da maior oferta de cursos e programas de pós-graduação, o que possibilita maior número de pesquisadores e estudos realizados.

Além disso, são necessários mais estudos para entender o porquê do interesse de orientadores e discentes em escrever uma dissertação de mestrado na área da fenomenologia, pois a utilização do complexo referencial teórico requer um tempo maior para reflexão e o mestrado dificulta essa possibilidade em razão dos prazos previstos para conclusão do curso. 
Tabela 3 - Caracterização do número de produções científicas segundo as regiões do Brasil, instituições e programas de pós-graduação. Brasil, 2003-2013

\begin{tabular}{|c|c|c|c|c|}
\hline Região & Instituição & Mestrado & Doutorado & Total \\
\hline \multirow{11}{*}{ Sudeste } & Universidade de São Paulo & 14 & 22 & 36 \\
\hline & Universidade Federal do Rio de Janeiro & 10 & 6 & 16 \\
\hline & $\begin{array}{l}\text { Universidade Federal do Estado } \\
\text { do Rio de Janeiro (UNIRIO) }\end{array}$ & 9 & - & 9 \\
\hline & Universidade Federal de Minas Gerais & 6 & - & 6 \\
\hline & Universidade do Rio de Janeiro & 4 & - & 4 \\
\hline & Universidade do Estado do Rio de Janeiro & 3 & - & 3 \\
\hline & Universidade Federal de São Paulo & 2 & - & 2 \\
\hline & Universidade de Guarulhos & 1 & - & 1 \\
\hline & Universidade Federal de Juiz de Fora & 1 & - & 1 \\
\hline & Universidade Estadual Paulista & - & 1 & 1 \\
\hline & Total & 50 & 29 & 79 \\
\hline \multirow{6}{*}{ Sul } & Universidade Estadual de Maringá & 5 & - & 5 \\
\hline & Universidade Federal de Santa Catarina & 1 & 4 & 5 \\
\hline & Universidade Federal de Santa Maria & 4 & - & 4 \\
\hline & Universidade Federal do Paraná & 3 & - & 3 \\
\hline & Universidade Estadual de Londrina & 1 & - & 1 \\
\hline & Total & 14 & 04 & 18 \\
\hline \multirow{6}{*}{ Nordeste } & Universidade Federal da Bahia & 3 & 1 & 4 \\
\hline & Universidade Federal do Ceará & - & 1 & 1 \\
\hline & Universidade de Pernambuco & 1 & - & 1 \\
\hline & Universidade Federal do Piauí & 1 & - & 1 \\
\hline & Universidade Estadual do Ceará & 1 & - & 1 \\
\hline & Total & 6 & 2 & 8 \\
\hline \multirow{3}{*}{$\begin{array}{l}\text { Centro- } \\
\text { Oeste }\end{array}$} & Universidade Federal de Goiás & 1 & - & 1 \\
\hline & Universidade Federal do Mato Grosso & 1 & - & 1 \\
\hline & Total & 2 & - & 2 \\
\hline \multirow{2}{*}{ Norte } & Universidade do Estado do Pará & 1 & - & 1 \\
\hline & Total & 1 & - & 1 \\
\hline
\end{tabular}

Fonte: Elaboração própria.

Nota: Sinal convencional utilizado:

- Dado numérico igual a zero não resultante de arredondamento.

\section{Considerações Finais}

De maneira geral, a pesquisa realizada identificou a significativa e importante valorização da área da enfermagem quanto ao referencial filosófico de Alfred Schutz, expresso pelo número de estudos voltados para o cuidado fundamentados nesse filósofo. Concluímos que o quantitativo das publicações gerado pela pós-graduação reflete o interesse pelo referencial teórico, assim como a busca profissional pelo aprimoramento do entendimento do ser por meio dos postulados fenomenológicos.

A menor disponibilização desses estudos, desenvolvidos em algumas regiões, pode ser explicada tanto pelo número inferior de cursos de pós-graduação stricto sensu quanto pela valorização e dificuldade de fomento para pesquisas 
com essa abordagem, dentre alguns dos motivos que perpassam a escolha da trajetória metodológica para o desenvolvimento de uma pesquisa.

Já no Sudeste do Brasil, a maior disponibilização desses estudos contribui para que a região se destaque em relação à produção científica sobre enfermagem e fenomenologia, com ênfase nos estudos oriundos de programas vinculados à Universidade de São Paulo e à Universidade Federal do Rio de Janeiro.

Diante da constatação da redução proporcional do número de pesquisas que envolvam abordagem fenomenológica ao longo dos anos, ressaltamos a importância de incentivar outras regiões para realizarem pesquisas dessa natureza, de modo a favorecer a investigação de novos paradigmas para o cuidar em Enfermagem. Tal estímulo poderá ser promovido, por exemplo, por meio da realização de eventos que visem divulgar a produção científica com abordagem fenomenológica e fomentar discussões a respeito do tema, evidenciando sua importância para a prática da enfermagem. Essa proposta suscita a reflexão crítica do profissional, a apreensão de novos conhecimentos e a percepção dos fenômenos vivenciados de formas alternativas.

Acreditamos ser de grande importância o desenvolvimento de estudos que utilizam como base os resumos publicados no banco de teses do CEPEn, em vista de sua credibilidade acadêmica. Entretanto, é necessário primar pela divulgação de todas as informações, incluindo o referencial filosófico utilizado, pois, por meio desse e de outros indicadores, será possível contribuir para a promoção e o fomento de novos estudos nessa área.

Uma vez que o conhecimento científico embasa e fornece subsídios para o cuidar e, por meio de estudos fenomenológicos, é possível construir novos horizontes no processo do cuidar, consideramos necessárias outras investigações, conduzidas sob esse referencial, de forma mais expressiva, para o progresso contínuo da profissão.

\section{Referências}

1. Duarte MR, Rocha SS. As contribuições da filosofia heideggeriana nas pesquisas sobre o cuidado em enfermagem. Cogitare enferm. 2011 abr/ jun;16(2):361-4.

2. Capalbo C. Abordando a enfermagem a partir da fenomenologia. Rev enferm UERJ. 1994;2(1): 70-6.

3. Ayres JRRM. O cuidado, os modos de ser (do) humano e as práticas de saúde. Saúde soc. 2004 set/dez;13(3):16-29.

4. Capalbo C. Alternativas metodológicas de pesquisa. Anais do $3^{\circ}$ Seminário Nacional de Pesquisa em Enfermagem; 1984 abr 3-6; Florianópolis (SC), Brasil. Florianópolis (SC): Universidade Federal de Santa Catarina; 1984. p. 130-57.

5. Merighi MAB. Fenomenologia. In: Merighi MAB, Praça NS. Abordagens teórico-metodológicas qualitativas: a vivência da mulher no período reprodutivo. Rio de Janeiro: Guanabara Koogan; 2003. p. 31-9.

6. Boemer MR. A condução de estudos segundo a metodologia de investigação fenomenológica. Rev latino-am enferm. 1994 jan;2(1):83-94.

7. Boemer MR, Rocha SMM. A pesquisa em enfermagem: notas de ordem histórica e metodológica. Saúde soc. 1996;5(2):77-88.

8. Salimena AMO, Dias BF, Mutti CF, Crivaro ET, Almeida IS, Souza IEO. Abordagem fenomenológica na pesquisa acadêmica de enfermagem: re-visitando a produção. Anais do $3^{\circ}$ Seminário Internacional de Pesquisa e Estudos Qualitativos; 2006 jun 1-3; São Bernardo do Campo (SP), Brasil. São Bernardo do Campo (SP): SE\&PQ; 2006.

9. Popim RC, Boemer MR. Cuidar em oncologia na perspectiva de Alfred Schütz. Rev latino-am enferm. 2005 set-out;13(5):677-85.

10. Heidegger M. Ser e tempo. Petrópolis: Vozes; 2012.

11. Jesus MCP, Capalbo C, Merighi MAB, Oliveira DM, Tocantins FR, Rodrigues BMRD, et al. A fenomenologia social de Alfred Schütz e sua contribuição para a enfermagem. Rev esc enferm USP [Internet]. 2013 June [citado 2016 abr 7];47(3):736-41. Disponível em: http://www. scielo.br/scielo.php?script=sci_arttext\&pid=S008062342013000300736\&lng=en.http://dx.doi. org/10.1590/S0080-623420130000300030.

Artigo apresentado em: 14/11/2015

Aprovado em: 8/4/2016

Versão final apresentada em: 4/6/2016 